\title{
Enzimotipagem de espécies do gênero Candida isoladas da cavidade bucal
}

\author{
Enzymotiping of species of the genus Candida \\ isolated from the oral cavity
}

\author{
Regina Celia Candido', Rosa Vitória Palamin Azevedo¹ e Marilena Chinalli Komesu²
}

\begin{abstract}
Resumo Foram avaliadas quanto a produção de exoenzimas fosfolipase e proteinase, 79 amostras de Candida isoladas da cavidade bucal de pacientes com lesões bucais características de candidose e indivíduos com boca clinicamente normal, atendidos na Faculdade de Odontologia de Ribeirão Preto USP. Dentre as cepas de C. albicans isoladas de lesões bucais, a fosfolipase e proteinase foram detectadas em, respectivamente, $83,3 \%$ e $66,7 \%$. C. tropicalis e C. parapsilosis produziram somente proteinase. Quanto às cepas isoladas de nichos sem lesão, do total de 32 C. albicans, $71,9 \%$ apresentaram fosfolipase e $68,7 \%$ proteinase. C. tropicalis apresentou apenas a enzima proteinase, C. glabrata, C. krusei, C. guilliermondii e Candida spp, não apresentaram nenhuma das exoenzimas. Entre as amostras de C. albicans de ambos os grupos, o enzimotipo 22 (fosfolipase positiva e proteinase fracamente positiva) foi prevalente. Enzimotipos diferentes foram detectados em amostras da mesma espécie provenientes de mesmo paciente.
\end{abstract}

Palavras-chaves: Leveduras. C. albicans. Enzimotipagem. Candidose bucal.

\begin{abstract}
The production of phospholipase and proteinase exoenzymes was evaluated in seventy nine samples of Candida isolated from the oral cavity of patients with oral lesions characteristic of candidosis and from individuals presenting a clinically normal mouth, attended at the University of Dentistry of Ribeirão Preto USP. Among the strains of C. albicans isolated from oral lesions, the phospholipase and proteinase were detected in $83.3 \%$ and $66.7 \%$, respectively. C. tropicalis and C. parapsilosis produced only proteinase. Regarding the isolated strains from niches without lesions, out of a total of $32 \mathrm{C}$. albicans, $71.9 \%$ presented phospholipase and $68.7 \%$ proteinase. C. tropicalis only presented the enzyme proteinase, C. glabrata, C. krusei, C. guilliermondii and Candida spp did not present any of the exoenzymes. Among the samples of C. albicans from both groups, the enzymotype 22 (positive phospholipase and proteinase weakly positive), was prevalent. Different enzymotypes of the same species were detected in samples collected from the same patient.
\end{abstract}

Key-words: Yeasts. C. albicans. Enzimotyping. Oral candidosis.

\footnotetext{
1. Departamento de Análises Clínicas, Toxicológicas e Bromatológicas da Faculdade de Ciências Farnacêuticas de Ribeirão Preto da Universidade de São Paulo, Ribeirão Preto, SP, 2. Departamento de Estomatologia da Faculdade de Odontologia da Universidade de São Paulo, Ribeirão Preto, SP, Brasil.

Auxílio: FAPESP

Endereço para correspondência. Dr ${ }^{a}$ Regina Celia Candido. Dept ${ }^{\circ}$ de Análises Clínicas Toxicológicas e Bromatológicas. Faculdade de Ciências Farmacêuticas de Ribeirão Preto/USP. Av. do Café s/n, 14040-903 Ribeirão Preto, SP, Brasil.

Telefax: 5516 602-4163.

e-mail rcandido @gly.fcfrp.usp.br

Recebido para publicação em 13/4/98.
} 
A candidose bucal pode ser causada por diferentes espécies do gênero Candida entre elas, C. albicans, C. tropicalis, C. glabrata, C. krusei, C. parapsilosis, C. guilliermondii, bem como por espécies de outros gêneros ${ }^{7} 9$. A quantidade de leveduras na lesão é geralmente alta e, freqüentemente, mais de uma espécie é isolada; neste caso, o papel de determinada espécie na etiologia da doença é de difícil avaliação.

A habilidade em produzir enzimas hidrolíticas é considerado importante fator patogênico ${ }^{2}$. As principais enzimas consideradas como fatores de virulência, produzidas por leveduras do gênero Candida, são as proteinases, que hidrolisam ligações peptídicas, e as fosfolipases, que hidrolisam os fosfoglicerídeos ${ }^{56}$. Foi sugerido que esta propriedade poderia ser usada como um critério para a biotipagem de C. albicans ${ }^{1014}$.

$A$ atividade de proteinase é variável entre as espécies e cepas do gênero Candida. Ray et al ${ }^{11}$ e De Bernardis et $\mathrm{al}^{4}$, utilizando procedimentos em meios sólidos, não observaram atividade enzimática em C. krusei, C. kefyr, C. guilliermondii e C. glabrata. No entanto, com técnicas mais sensíveis como o SDS-PAGE, verificaram que C. kefyr e C. guilliermondii eram capazes de hidrolisar a albumina, e que $C$. glabrata e $C$. krusei eram destituídas de qualquer atividade proteolítica.

Williamson et $\mathrm{al}^{18}$, usando o método descrito por Price et al ${ }^{10}$ modificado, desenvolveram um novo sistema de tipagem para $C$. albicans, buscando detectar a atividade da enzima fosfolipase de 100 cepas isoladas a partir da cavidade bucal. Verificaram que $94 \%$ de $C$. albicans foram produtoras de fosfolipase, com graus de atividade variando com valores de $P Z$ de 0,3 a 0,9.

Delineamos este trabalho, tendo como objetivo a detecção de enzimas extracelulares fosfolipase e proteinase pelas espécies de leveduras isoladas da cavidade bucal de pacientes com e sem candidose bucal.

\section{MATERIAL E MÉTODOS}

Foram avaliados 50 indivíduos adultos entre os pacientes atendidos na Faculdade de Odontologia de Ribeirão Preto USP. Deles, 19 apresentavam boca clinicamente saudável (grupo controle) e 31 possuíam algum tipo de lesão intraoral, com suspeita clínica de candidose bucal, entre elas, estomatite protética, glossite rômbica mediana, queilite angular, língua fissurada.

Amostras do dorso da língua, lesão e saliva foram semeadas no ágar Sabouraud-dextrose com cloranfenicol, incubado a $37^{\circ} \mathrm{C}$, durante 3 a 5 dias. Após o isolamento, as leveduras foram identificadas seguindo a metodologia clássica ${ }^{17}$.

Setenta e nove cepas do gênero Candida, foram submetidas à prova de detecção de enzimas fosfolipase ${ }^{1018}$ e proteinase ${ }^{12}$. Volumes de aproximadamente $5 \mathrm{ml}$ de suspensões de cepas em água destilada esterilizada, equivalente a 1 da escala de MacFarland foram inoculadas em pontos eqüidistantes, respectivamente, nos meio de ágar fosfolipase e ágar proteinase. Os testes foram realizados em duplicata. As placas contendo 4 inóculos de diferentes cultivos, permaneceram incubadas a $37^{\circ} \mathrm{C}$, durante 4 dias para fosfolipase e 7 dias para proteinase.
A presença de enzima fosfolipase foi observada pela formação de uma zona opaca ao redor da colônia da levedura e a atividade enzimática (PZ) foi medida dividindo-se o diâmetro da colônia pelo diâmetro da colônia mais a zona de precipitação. PZ foi codificada com um dígito, com valores iguais a 1,2 ou 3 (enzimotipo), respectivamente, para as cepas não produtoras desta enzima $(P Z=1)$, para a atividade média $(P Z \geq 0,64<1,0)$ e para a atividade enzimática elevada ( $P Z \leq 0,63)$.

A presença da enzima proteinase foi observada pela formação de um halo transparente ao redor da colônia da levedura. Proteinase foi codificada com dígitos variando de 1 a 4, de acordo com o grau de atividade enzimática, ou seja, sem atividade ou negativa $=1$; fraca, halo menor que $1 \mathrm{~mm}=2$; média, halo entre $1 \mathrm{a} 2 \mathrm{~mm}=3$ e forte, halo maior que $2 \mathrm{~mm}=4$.

As cepas foram codificadas com algarismos contendo 2 dígitos representados, respectivamente, por fosfolipase e proteinase. O enzimotipo 11, representado as cepas fosfolipase e proteinase negativas e o enzimotipo 32 as amostras fosfolipase fortemente e proteinase fracamente positivas.

\section{RESULTADOS}

Verificamos que $C$. albicans foi a mais prevalente em todos os tipos de amostras examinadas. Entre os pacientes com lesões características de candidose bucal, outras espécies, também, foram isoladas, principalmente, de saliva e lesão, com uma freqüência maior para 
C. tropicalis e C. glabrata. C. guilliermondii e C. krusei foram detectadas somente de pacientes do grupo controle conforme representado na Tabela 1 , a maioria $(58,8 \%)$ das leveduras isoladas de lesão apresentaram atividade da enzima fosfolipase e proteinase.

Quanto às cepas isoladas de nichos sem lesão, verificamos que $23(51,1 \%)$ produziram fosfolipase e, $26(57,8 \%)$ proteinase; destacandose que $15(33,3 \%)$ apresentaram ambas enzimas estudadas (Tabela 1).

Em relação às espécies do gênero Candida isoladas, da cavidade bucal, com e sem candidose, respectivamente, $83,3 \%$ e $71,9 \%$ das $C$. albicans apresentaram atividade de fosfolipase e $66,7 \%$ (lesão) e 68,7\% (sem lesão) foram positivas para

Tabela 1 - Perfil da atividade enzimática das leveduras do gênero Candida, isoladas da cavidade bucal.

\begin{tabular}{|c|c|c|c|c|c|c|c|c|c|c|c|c|c|c|}
\hline \multirow{4}{*}{ Candida } & \multicolumn{14}{|c|}{ Atividade enzimática } \\
\hline & \multicolumn{4}{|c|}{ fosfolipase } & \multicolumn{4}{|c|}{ proteinase } & \multicolumn{6}{|c|}{ fosfolipase $\mathrm{x}$ proteinase } \\
\hline & \multicolumn{2}{|c|}{ negativa } & \multicolumn{2}{|c|}{ positiva } & \multicolumn{2}{|c|}{ negativa } & \multicolumn{2}{|c|}{ positiva } & \multicolumn{2}{|c|}{ nenhuma } & \multicolumn{2}{|c|}{ ambas } & \multicolumn{2}{|c|}{ uma } \\
\hline & $\mathrm{n}^{0}$ & $\%$ & $\mathrm{n}^{0}$ & $\%$ & $\mathrm{n}^{\circ}$ & $\%$ & $\mathrm{n}^{0}$ & $\%$ & $\mathrm{n}^{\circ}$ & $\%$ & $\mathrm{n}^{\circ}$ & $\%$ & $\mathrm{n}^{\circ}$ & $\%$ \\
\hline Com lesão (34) & 14 & 41,2 & 20 & 58,8 & 14 & 41,2 & 20 & 58,8 & 6 & 17,6 & 12 & 35,3 & 16 & 47,1 \\
\hline Sem lesão (45) & 22 & 48,8 & 23 & 51,1 & 19 & 42,2 & 26 & 57,8 & 11 & 24,4 & 15 & 33,3 & 19 & 42,2 \\
\hline
\end{tabular}

proteinase (Tabela 2). C. tropicalis, $C$. parapsilosis, C. glabrata e Candida spp não demonstraram produção de fosfolipase.
Proteinase foi detectada apenas nas amostras de $C$. tropicalis isoladas de ambos os grupos e de C. parapsilosis isolada de lesão (Tabela 2).

Tabela 2 - Atividade enzimática das diferentes espécies do gênero Candida isoladas de pacientes com e sem lesão bucal.

\begin{tabular}{|c|c|c|c|c|c|c|c|c|c|c|}
\hline \multirow{3}{*}{ Espécies } & \multicolumn{5}{|c|}{ Com lesão } & \multicolumn{5}{|c|}{ Sem lesão } \\
\hline & \multirow{2}{*}{$\begin{array}{c}\text { cepas } \\
n^{\circ}\end{array}$} & \multicolumn{2}{|c|}{ fosfolipase } & \multicolumn{2}{|c|}{ proteinase } & \multirow{2}{*}{$\begin{array}{c}\text { cepas } \\
n^{0}\end{array}$} & \multicolumn{2}{|c|}{ fosfolipase } & \multicolumn{2}{|c|}{ proteinase } \\
\hline & & no & $\%$ & $\mathrm{n}^{\circ}$ & $\%$ & & $\mathrm{n}^{\circ}$ & $\%$ & $\mathrm{n}^{0}$ & $\%$ \\
\hline C. albicans & 24 & 20 & 83,3 & 16 & 66,7 & 32 & 23 & 71,9 & 22 & 68,7 \\
\hline C. tropicalis & 5 & 0 & 0 & 3 & 60 & 6 & 0 & 0 & 4 & 66,7 \\
\hline C. glabrata & 3 & 0 & 0 & 0 & 0 & 2 & 0 & 0 & 0 & 0 \\
\hline C. parapsilosis & 1 & 0 & 0 & 1 & 100 & 1 & 0 & 0 & 0 & 0 \\
\hline C. guilliermondii & 0 & 0 & 0 & 0 & 0 & 1 & 0 & 0 & 0 & 0 \\
\hline C. krusei & 0 & 0 & 0 & 0 & 0 & 2 & 0 & 0 & 0 & 0 \\
\hline Candida spp & 1 & 0 & 0 & 0 & 0 & 1 & 0 & 0 & 0 & 0 \\
\hline Total & 34 & 20 & & 19 & & 45 & 23 & & 24 & \\
\hline
\end{tabular}

Conforme apresentado na Tabela 3, foram detectados 10 diferentes enzimotipos, com prevalência do 11 representando as 17 amostras negativas para a produção de fosfolipase e proteinase, sendo 2 C. albicans e as demais Candida spp. O segundo enzimotipo foi o 22 representando as amostras fosfolipase positivas e proteinase fracamente positivas, este foi detectado somente em 16 C. albicans. O enzimotipo 14 (fosfolipase negativa e proteinase fortemente positiva) foi verificado em apenas 1 C. parapsilosis isolada de lesão. Os demais enzimotipos foram encontrados com freqüência variável.

Em relação aos 4 pacientes que apresentavam lesões múltiplas (Tabela 4), observamos que eram causadas por cepas da mesma ou de espécies diferentes, sendo que as espécies iguais apresentaram enzimotipos iguais somente em uma ocasião (paciente 19). 
Tabela 3 - Distribuição dos enzimotipos de espécies de Candida isolados de lesão e de outros nichos da cavidade bucal.

\begin{tabular}{|c|c|c|c|c|c|c|}
\hline \multirow[b]{2}{*}{ Enzimotipos } & \multicolumn{2}{|c|}{ C. albicans } & \multicolumn{2}{|c|}{ C. tropicalis } & \multicolumn{2}{|c|}{ Candida spp* } \\
\hline & lesão & outros nichos & lesão & outros nichos & lesão & outros nichos \\
\hline 11 & & 2 & 2 & 2 & 4 & 7 \\
\hline 12 & 3 & 5 & 1 & 3 & & \\
\hline 13 & 1 & 2 & 2 & 1 & & \\
\hline 14 & & & & & 1 & \\
\hline 21 & 5 & 5 & & & & \\
\hline 22 & 7 & 9 & & & & \\
\hline 23 & 1 & & & & & \\
\hline 31 & 3 & 3 & & & & \\
\hline 32 & 4 & 5 & & & & \\
\hline 33 & & 1 & & & & \\
\hline Total & 24 & 32 & 5 & 6 & 5 & 7 \\
\hline
\end{tabular}

* C. guilliermondii; C. krusei; C. glabrata; C. parapsilosis; Candida spp

Tabela 4 - Distribuição dos enzimotipos de leveduras do gênero Candida, isoladas de pacientes com múltiplas lesões de candidose bucal.

\begin{tabular}{|c|c|c|c|c|}
\hline Paciente & $\begin{array}{c}\text { Cepa } \\
\left(n^{\circ}\right)\end{array}$ & Espécie & Tipo de lesão & Enzimotipo \\
\hline \multirow[t]{4}{*}{19} & 19.1 & C. tropicalis & estomatite protética & 13 \\
\hline & 19.2 & C. albicans & glossite rômbica mediana & 31 \\
\hline & 19.3 & C. tropicalis & glossite rômbica mediana & 11 \\
\hline & 19.4 & C. tropicalis & queilite angular & 13 \\
\hline \multirow[t]{2}{*}{20} & 20.2 & C. albicans & estomatite protética & 22 \\
\hline & 20.1 & C. albicans & língua fissurada & 21 \\
\hline \multirow[t]{2}{*}{25} & 25.2 & C. glabrata & língua fissurada & 11 \\
\hline & 25.1 & C. albicans & glossite rômbica mediana & 22 \\
\hline \multirow[t]{2}{*}{39} & 39.3 & C. albicans & estomatite protética & 12 \\
\hline & 39.1 & C. albicans & glossite rômbica mediana & 22 \\
\hline
\end{tabular}

\section{DISCUSSÃO}

A detecção das enzimas fosfolipase e proteinase em meios sólidos tem sido descrita, principalmente, em amostras de C. albicans.

Quanto ao fato de termos detectado a produção de fosfolipase, somente pelas cepas pertencentes à espécie $C$. albicans, concorda com resultados obtidos por alguns pesquisadores, tal como Samaranayake et $\mathrm{al}^{14}$, que estudaram a atividade fosfolipásica de 41 cepas de leveduras do gênero Candida e observaram que este tipo de enzima era produzida por $C$. albicans, mas, não por $C$. tropicalis, $C$. glabrata e $C$. parapsilosis. No entanto, Shimizu ${ }^{15}$ verificou que, além de C. albicans e C. parapsilosis, os cultivos de C. tropicalis, C. guilliermondii e C. krusei, também, evidenciavam atividade e fosfolipase, só que em diferentes percentuais.

Estudos anteriores têm mostrado que 30 a $100 \%$ da cepas de C. albicans são secretoras de fosfolipase, com graus variáveis de atividade ${ }^{101415}$ No Brasil, Maffei ${ }^{8}$ verificou que $51,1 \%$ das cepas estudadas apresentavam esta enzima; no estudo de Souza et $\mathrm{al}^{16}$, este percentual atingiu $73,3 \%$; no de Candido ${ }^{3}, 96,6 \%$ e Shimizu ${ }^{15}$ verificou que $100 \%$ das C. albicans apresentavam atividade para fosfolipase.

Nossos resultados, em relação à atividade fosfolipásica de $C$. albicans, com graus variáveis de atividade, estão dentro dos parâmetros encontrados por autores estrangeiros ${ }^{10} 14$. Comparando com resultados de pesquisadores brasileiros, nosso percentual foi maior que o obtido por Maffei ${ }^{8}$ e Souza et $a l^{16}$. No entanto, foi bem menor que o de Candido ${ }^{3}(96,6 \%)$ e Shimizu ${ }^{15}$ $(100 \%)$; o que pode estar relacionado com diversidade de amostragem, principalmente.

Quanto à produção de exoenzimas, pelas leveduras isoladas de lesão bucal, observamos 
que $58,8 \%$ apresentavam atividade fosfolipásica e proteolítica, ressaltando-se que $35,3 \%$ das cepas testadas demonstraram os dois tipos de atividade enzimática; valores semelhantes aos obtidos com leveduras isoladas de nichos sem lesão, indicando a importância de se efetuar uma correlação entre a produção dessas enzimas e a sua patogenicidade, principalmente se isoladas de indivíduos considerados clinicamente sadios, para se adotar métodos preventivos mais acurados.

Quanto às espécies isoladas de lesão, observamos que a maior parte das $C$. albicans apresentava atividade enzimática, fosfolipase $(83,3 \%)$ e proteinase $(66,7 \%)$. Em relação à $C$. tropicalis, (3/60\%) produziram proteinase e, $50 \%$ de C. parapsilosis (2), também deram resultado positivo para esta prova, demonstrando ser um dos prováveis fatores de virulência deste gênero.

Nossos resultados quanto à detecção de atividade proteolítica foram menores, dos obtidos por Yamamoto et al ${ }^{19}$, em relação à $C$. tropicalis, C. parapsilosis e C. guilliermondii.

Especificamente, em relação aos dados obtidos com as espécies $C$. glabrata e $C$. krusei, concordam com os de outros pesquisadores ${ }^{41119}$, que também não observaram atividade proteolítica, se bem que Banerjee et al ${ }^{1}$ admitam que C. glabrata produza pequena quantidade de proteinase e que não seja excretada.

Analisando-se o grau de atividade enzimática, na detecção de proteinase, verificamos que nossos resultados foram diferentes dos obtidos por Rüchel et $\mathrm{al}^{13}$. Esta divergência poderá estar relacionada com o sítio de amostragem; se tem a ver com casos de candidose e com o hospedeiro examinado. Ressaltamos que, em nosso estudo, os resultados obtidos entre as espécies foram diferentes, contrastando com a semelhança observada pelos autores, entre $C$. albicans e $C$. tropicalis.

Verificamos que foram detectados enzimotipos diferentes, entre cepas isoladas em lesões diferentes, do mesmo paciente. Na maioria das vezes, o padrão indica que pelo menos uma ou ambas as enzimas foram produzidas, comprovando a provável patogenicidade da cepa envolvida, além de ajudar na discriminação, como é o caso do paciente 19, com as cepas detectadas na queilite angular; podendo, portanto, servir como um marcador epidemiológico.

\section{REFERÊNCIAS BIBLIOGRÁFICAS}

1. Banerjee A, Ganesan K, Datta A. Induction of secretory acid proteinase in Candida albicans. Journal of General Microbiology 137:2455-2461, 1991.

2. Barrett-Bee K, Hayes Y, Wilson RG, Ryley JF. A comparison of phospholipase activity, cellular adherence and pathogenicity of yeasts. Journal of General Microbiology 131:1217-1221, 1985.

3. Candido RC. Candida albicans: marcadores epidemiológicos em amostras isoladas de diferentes materiais biológicos. Tese de Doutorado, Universidade Federal de São Paulo, São Paulo, SP, 1991.

4. De Bernardis F, Chiani P, Ciccozzi M, Pellegrini G, Ceddia T, D'Offizzi G, Quinti I, Sullivan PA, Cassone A. Elevated aspartic proteinase secretion and experimental pathogenicity of Candida albicans isolates from oral cavities of subjects infected with Human Immunodeficiency Virus. Infectious Immunity 64:446-471, 1996.

5. Ghannoum M, Abu-Elteen $\mathrm{KH}$. Correlative relationship between proteinase production, adherence and pathogenicity of various strains of Candida albicans. Journal Medical and Veterinary Mycology 24:407-413, 1986.

6. Ghannoum MA, Abu-Elteen KH. Pathogenicity determinants of Candida. Mycoses 33:265-282, 1990.

7. Kolnick JR. Oral candidosis. Report of case implicating Candida parapsilosis as a pathogen. Oral Surgery, Oral
Medicine, Oral Pathology, Oral Radiology and Endodontics 50:411-415, 1980.

8. Maffei CML. Amostras de Candida albicans isoladas de gestantes: fatores de virulência, sensibilidade a antifúngicos, tipagem fenotípica e genotípica. Tese de Doutorado, Universidade de São Paulo. São Paulo, 1996.

9. Odds FC. Pathogenesis of Candida infections. Journal American Academic Dermatology 31:S2-S5, 1994.

10. Price MF, Wilkinson ID, Gentry IO. Plate method for detection of phospholipase activity in Candida albicans. Sabouraudia 20:15-20, 1982.

11. Ray TL, Payne CD. Candida albicans acid proteinase: a role in virulence. In: Ayoub EM, Cassel GH, Branche WC, Henry TJ (eds) Microbiology determinants of virulence and host response. American Society of Microbiology, Washington, p.163-178, 1990.

12. Rüchel R, Tegeler R, Trost M. A comparison of secretory proteinases from different strains of Candida albicans. Sabouraudia 20:233-244, 1982.

13. Rüchel R, Uhlemann K, Böning B. Secretion of acid proteinases by different species of the genus Candida. Zentralblatt für Bakteriologie und Hygiene, I. Abteilung Originale A 255:537-548, 1983.

14. Samaranayake LP, Raeside JM, Mcfarlane TW. Factors affecting the phospholipase activity of Candida species in vitro. Sabouraudia 22:201-207, 1984. 
15. Shimizu MT. Fosfolipase em espécies de Candida. Revista de Microbiologia 20:338, 1989.

16. Souza EMB, Paula CR, Purchio A, Gambale W, Corrêa $B$, Cury AE. Aspectos morfo-fisiológicos, fatores de virulência e sensibilidade à antifúngicos de amostras de Candida albicans, sorotipos A e B, isoladas em São Paulo, Brasil. Revista de Microbiologia 21:247-253, 1990.

17. Van der Walt JP, Yarrow D. Methods for the maintenance, classification and identification of yeast. In: Kreger Van Rij NJW (eds) The yeasts: a taxonomic study. Amsterdam, Elsevier, p.45-104, 1984
18. Williamson MI, Samaranayake LP, Mac Farlane TW. Phospholipase activity as a criterion for biotyping Candida albicans. Journal Medical and Veterinary Mycology 24:415-417, 1986.

19. Yamamoto T, Nohara K, Uchida K, Yamaguchi H. Purification and characterization of secretory proteinase. Microbiology and Immunology 36:637-641, 1992.

20. Zegarelli D. Fungal infections of the oral cavity. Otolaryngologic Clinics of North America 26:1069-1088, 1993. 